Rev. Elev. Méd. vét. Pays trop., 1974, 27 (3) : 285-299

\title{
Contribution à l'étude de la répartition des tiques en Éthiopie (enquête effectuée de 1965 à 1969)
}

\author{
par P. BERGEON $\left(^{*}\right)$ et J. BALIS $\left({ }^{* *}\right)$
}

\begin{abstract}
RESUME
Les auteurs ont étudié la répartition des tiques en Ethiopie et quarante espèces ont été inventoriées; Rhipicephalus pulchellus, Amblyomma variegatum, Amblyomma cohaerens et Rhipicephalus simus simus sont numériquement les plus importantes. Amblyomma variegatum est l'espèce la plus répandue.
\end{abstract}

Pendant longtemps le cheptel éthiopien paya un très lourd tribut aux grandes épizooties. Ces dernières, grâce à des mesures de prophylaxie sanitaires et médicales, sont actuellement bien contrôlées; mais d'autres causes de mortalité telles que la cowdriose, les piroplasmoses et les theilérioses, considérées autrefois comme secondaires, prennent une place d'autant moins négligeable que les rassemblements d'animaux sont plus importants. Le rôle bien connu de vecteur actif joué par les tiques justifie l'étude de la fréquence et de la répartition de ces acariens en Ethiopie. Ce travail devrait, dans une certaine mesure, en précisant les espèces et leurs zones d'action, permettre ultérieurement la mise en cuvre de moyens efficaces de lutte.

(*) « La Quieta », 49, rue Sous Barri, 06800 Cagnessur-Mer.

(**) Laboratoire de Debré-Zeit, Institut Impérial Vétérinaire, P.O. Box 19, Debré-Zeit, Ethiopie.

N.D.L.R. : Le texte présenté correspond à l'avancement de l'étude sur les tiques d'Ethiopie et aux conceptions de l'auteur principal à la fin de l'année 1969, époque où il dut interrompre ses activités pour raison de santé. Devant l'intérêt de ce travail, il a semblé préférable de le publier tel quel, même avec un retard de plusieurs années, sans y pratiquer de corrections ou de mises à jour.

\section{Travaux antérieurs}

Les tiques d'Ethiopie sont surtout connues depuis les travaux des auteurs italiens, travaux résumés dans une excellente publication d'Emilia STELLA accompagnée d'une importante bibliographie (7).

En 1938, cet auteur avait recensé sur tout le territoire, à l'exception de la Somalie, les 15 espèces suivantes :

Genre Amblyomma :
A. hebraeum
A. marmoreum
A. rugosum
A. schlottkei
A. variegatum

Genre Dermacentor :
D. reticulatus

Genre Haemaphysalis:

H. leachii

Genre Hyalomma:

H. aegyptium impressum

H. erythraeum

Genre Margaropus :

$M$. decoloratus 
Genre Rhipicephalus :
$R$. appendiculatus
$R$. bursa
$R$. oculatus
R. pulchellus
$R$. sanguineus
R. simus

A cette liste il faut ajouter 3 espèces de la famille des Argasidae:

Argas persicus

Ornithodoros moubata

Ornithodoros savignyi

toutes trois récoltées seulement en Erythrée.

\section{Travaux personnels}

Ils ont été entrepris en 1965 et poursuivis d'année en année jusqu'en fin 1969. Ils avaient déjà fait l'objet, en 1968, d'un rapport au gouvernement éthiopien (1). Les collectes furent effectuées soit par nous-mêmes soit par des confrères éthiopiens, français ou étrangers aidés par le personnel assistant et vaccinateur du Service Vétérinaire Impérial. Nous tenons à remercier pour leur collaboration les Docteurs vétérinaires Assefa Wolde Georgis (Arussi), Blanc, chef de la Mission Vétérinaire Française jusqu'en 1971 (Borana), Colson (Gemu Gofa et Sidamo), Firke (Godjam), Girma Tadegge (Gemu Gofa), Gratteau (Gemu Gofa et Sidamo), Kovalenko (Bale et Wollega), Laurent (Arussi et Sidamo), Lernould (Bale), Tager Kagan (Gemu Gofa). Nous remercions également la Mission Vétérinaire Chinoise (Taipeh) pour son excellente coopération dans la province de Begemder, ainsi que Messieurs Getahoun Tebedje, Guebre Negus Tasfa Mariam et Morkuria Worku dont nous avons apprécié l'aide technique.

Nous nous sommes référés aux publications de TOUMANOFF (8), WALKER $(9,10,11$, 12), MOREL et VASSILIADES (5), ROUSSELOT (6), mais surtout à celles dHOOGSTRAAL $(2,3,4)$. Ce dernier auteur a suivi nos travaux et a bien voulu déterminer luimême des échantillons en litige, notamment des Rhipicephalus tels que $R$. appendiculatus dont l'existence était mise en doute par certains.

Les tiques furent avant tout prélevées sur le bétail, ce qui confère donc à cet inventaire un intérêt pratique.

Bien que portant sur 10246 échantillons récoltés et identifiés, cette étude est cependant incomplète en raison de la superficie tourmentée du territoire et il serait de plus nécessaire de l'étendre aux animaux sauvages et aux oiseaux.

Les 40 espèces inventoriées ont été classées par province. Douze d'entre elles font l'objet de petites cartes de répartition sur lesquelles, par soucis de simplification, ne figurent que le tracé des frontières provinciales et les lieux de récolte; on peut identifier ces éléments en consultant la carte à plus grande échelle où sont inscrits les noms, des localités et provinces, cités dans le textc.

\section{INVENTAIRE DES RECOLTES}

\section{$A R G A S I D A E$}

Le nombre restreint d'exemplaires recueillis (9 mâles, 1 femelle) ne peut donner une idée exacte de la répartition de ces acariens.

Genre Argas :

A. persicus, Shoa, Debre-Zeit 12-65, poule, $2 \mathrm{~m} ., 1 \mathrm{f}$.

\section{Genre Ornithodoros:}

O. moubata: Begemder, Gondar, 6-67, sol d'habitation, $4 \mathrm{~m}$.

O. savignyi : Erythree, Asmara, 4-65, zébu, $3 \mathrm{~m}$.

\section{IXODIDAE}

Nous avons pu dénombrer 37 espèces réparties en 6 genres.

Genre Amblyomma : 3373 mâles, 1099 femelles, 7 nymphes.

A. cohaerens (Dönitz, 1909), 1076 mâles, 523 femelles (carte).

C'est une espèce dominante dans le sud-ouest de l'Ethiopie; c'est ainsi par exemple que $88 \mathrm{p}$. 100 des tiques récoltées à Séghé, dans le Wollega, étaient des $A$. cohaerens. Cette particulière abondance est peut-être due au fait que les buffles étaient autrefois fréquents dans cette province. Il semble cependant que cette espèce soit dintroduction relativement récente car Emilia STELLA ne la mentionne pas en 1938 et HOOGSTRAAL, dans une correspondance personnelle, précise qu'avant 1956 personne ne la considérait comme très commune dans le pays. 


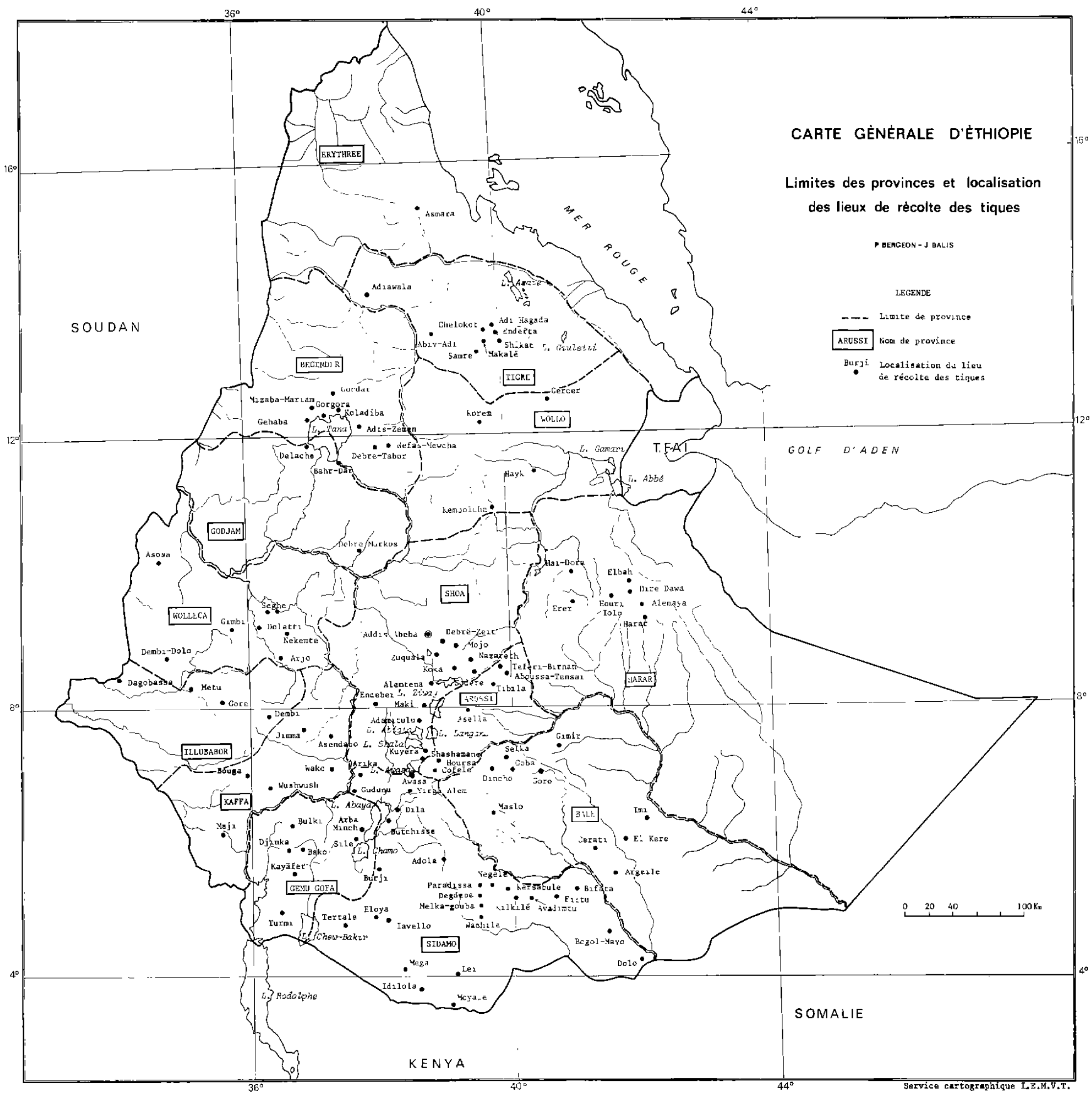


箅
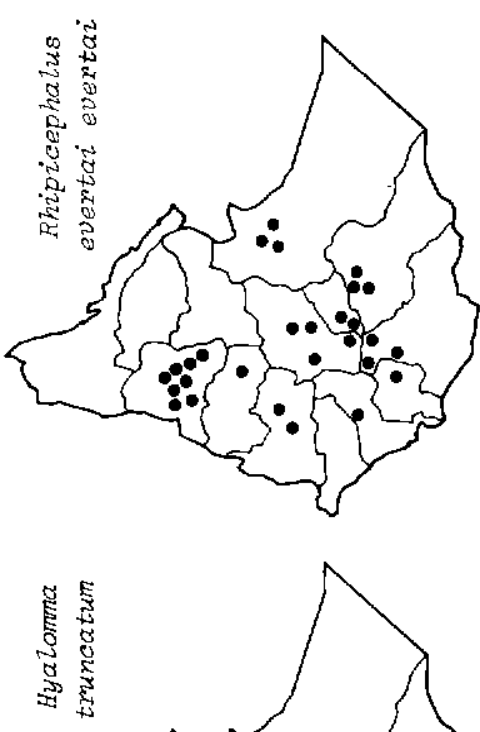

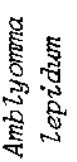

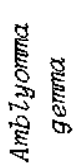

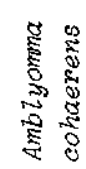
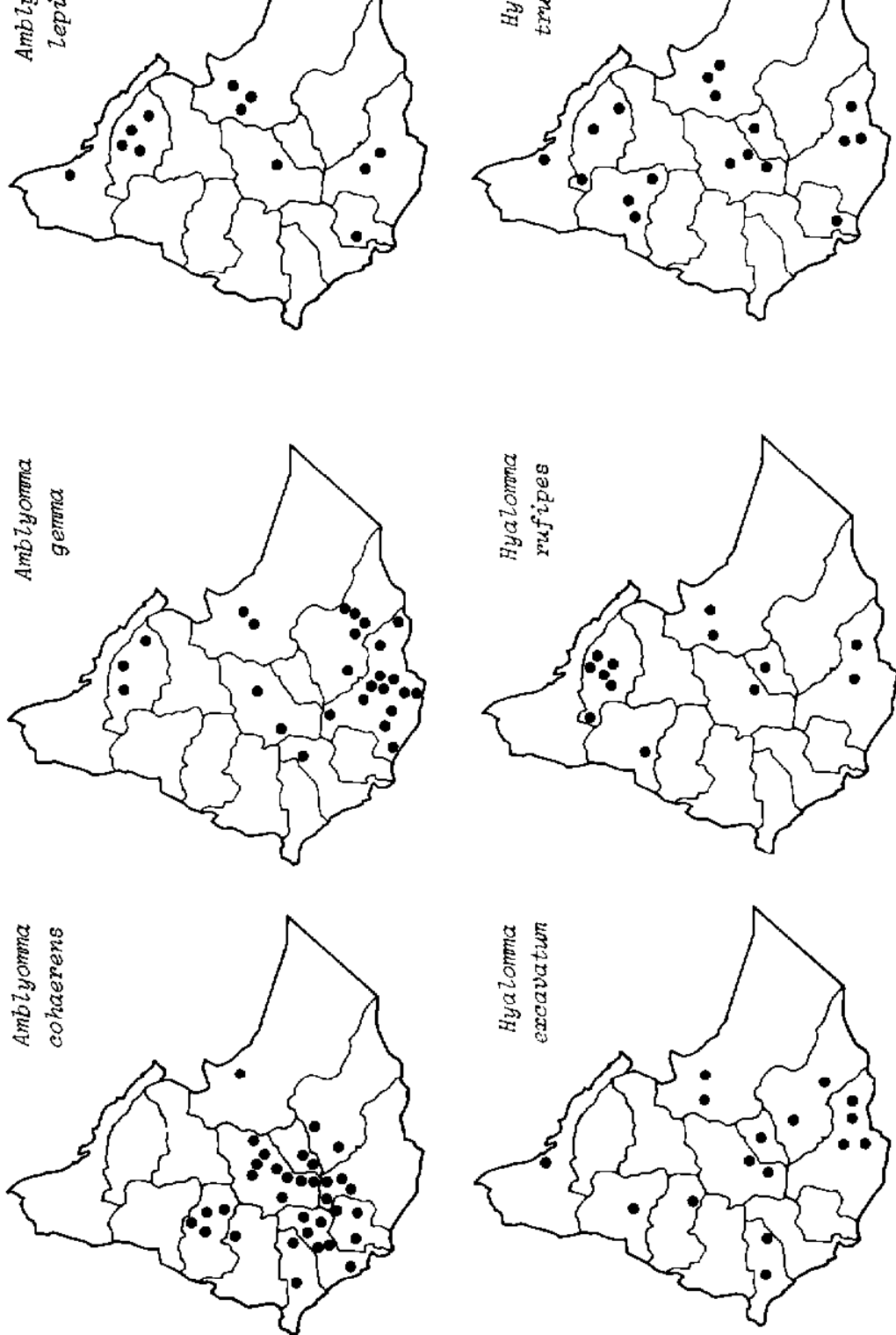
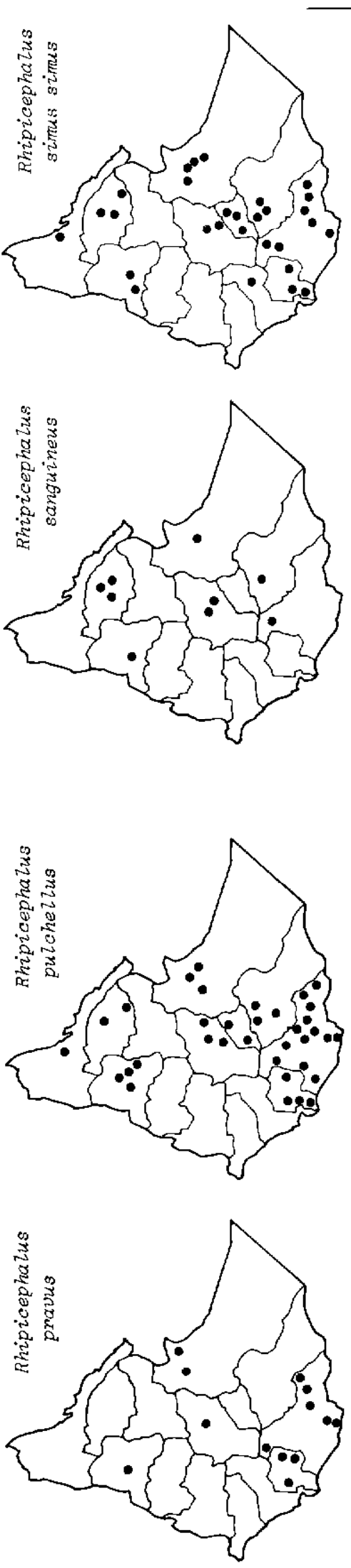
Nous avons trouvé cette tique dans 10 provinces.

\section{- ARUSSI :}

Cofele, 6-68, cheval, $2 \mathrm{~m}$.

Hoursa (2 $800 \mathrm{~m}), 6-66$, mouton, $1 \mathrm{f}$., zébu, $114 \mathrm{~m} ., 37 \mathrm{f}$.

\section{- BALE :}

Goro (1 $800 \mathrm{~m}), 10-67$, zébu, 1 m., 2 f.; 9-68, zébu, 13 m., 7 f.

Maslo, 8-68, zébu, 90 m., 30 f.

\section{- GEMU GOFA :}

Djinka, 5-68, zébu, $5 \mathrm{~m}$.

Sile, 5-69, zébu, $21 \mathrm{~m}$., $11 \mathrm{f}$.

- GODJAM :

Bahr Dar, 6-67, zébu, 1 m., 1 f.

- HARAR :

Dire Dawa, 6-67, zébu, 2 m, 1 f.

\section{- ILLUBABOR :}

Dembi, 11-66, zébu, $20 \mathrm{~m}$.

Gore, 9-66, zébu, 1 m., 1 f.

\section{- KAFFA :}

Assendabo, 4-66, zébu, 17 m., 7 f.

Bonga, 9-68, zébu, $50 \mathrm{~m}, 36 \mathrm{f}$.

Jimma, 1-66, zébu, $73 \mathrm{~m}$., $41 \mathrm{f}$.

Maji, 4-66, zébu, $10 \mathrm{~m} ., 8 \mathrm{f}$.

Wako, 4-66, zébu, 12 m., 25 f.

Wushwush, 4-66, zébu, $9 \mathrm{~m}$.

\section{- SHOA :}

Addis-Abeba, 12-67, zébu, 26 m., 28 f.; 5-68, zébu, 18 m., 27 f.

Alem Tena, 4-68, zébu, $4 \mathrm{~m} ., 2 \mathrm{f}$.

Awash, 4-69, zébu, 24 m., 5 f.

Debre Zeit, 5-66, zébu, $6 \mathrm{~m}$.

Endeber, 5-67, âne, 4 m., 1 f., cheval, 1 f., zébu, $62 \mathrm{~m}$., $10 \mathrm{f}$.

Kuyera, 12-67, zébu, 81 m., 43 f.; 4-69, zébu, $10 \mathrm{~m}$., $12 \mathrm{f}$.

Maki, 6-68, zébu, 9 m., 9 f.

Mojo, 12-67, zébu, $12 \mathrm{~m}$.

Shashamane, 5-66, zébu, 8 m., 20 f.; 7-67, zébu, $11 \mathrm{~m}$., 1 f.; 4-69, zébu, 51 m., 18 f.

- SIDAMO :

Arika (1600 m), 5-68, zébu, $1 \mathrm{~m}$.
Awasa, 2-66, zébu, $1 \mathrm{~m}$; 3-66, zébu, 5 m.; 11-66, zébu, 38 m., 29 f.; 4-69, zébu, 20 m., $7 \mathrm{f}$.

Butchissé, 2-66, zébu, $5 \mathrm{~m}$.

Gudunu (1700 m), 5-68, zébu, $3 \mathrm{~m} ., 3 \mathrm{f}$. Yrgalem (2000 m), 11-66, zébu, 38 m., 29 f;; 11-67, zébu, 26 m., 12 f.

\section{- WOLLEGA :}

Arjo $(3000 \mathrm{~m}), 5-65$, zébu, $38 \mathrm{~m} ., 12 \mathrm{f}$. Doletti, 12-65, zébu, $17 \mathrm{~m}, 5 \mathrm{f}$.

Nekemte (1900 m), 5-69, zébu, 51 m., 10 f. Séghé (1 $700 \mathrm{~m}), 5-65$, zébu, 64 m., 28 f.; 1-66, zébu, 5 m., 2 f.

A. eburneum (Gerstäcker, 1873), (identification confirmée par H. HOOGSTRAAL), 5 mâles.

\section{- HARAR :}

Dire Dawa, 7-66, zébu, $1 \mathrm{~m}$.

\section{- TIGRE :}

Kwiha, 4-66, zébu, $1 \mathrm{~m}$.

Shikat, 4-66, chèvre, $2 \mathrm{~m}$, mouton, $1 \mathrm{~m}$.

A. gemma (Dönitz, 1909), 245 mâles, 91 femelles, 2 nymphes (carte).

Quelques exemplaires de la province de Harar ont été identifiés par HOOGSTRAAL. Cette tique a été trouvée surtout dans le sud du pays.

\section{- BALE :}

Argeile, 7-68, zébu, $3 \mathrm{~m}$.

Cerati, 5-69, dromadaire, $7 \mathrm{~m}$.

El Kere, 5-69, zébu, $8 \mathrm{~m}$.

Imi, 7-68, zébu, $5 \mathrm{~m}, 1 \mathrm{f}$.

Maslo, 8-68, dromadaire, $8 \mathrm{~m}$., zébu, $21 \mathrm{~m}$, $2 \mathrm{f}$.

\section{- HARAR :}

Elbah, 7-66, mouton, $1 \mathrm{~m}$., zébu, $6 \mathrm{~m}$.

Houri Tolo $(800 \mathrm{~m}), 7-66$, zébu, $9 \mathrm{~m}$

- KAFFA :

Jimma, 7-66, zébu, $3 \mathrm{~m}$.

- SHOA :

Debre Zeit, 10-66, zébu, $1 \mathrm{~m}$.

Endeber, 5-67, zébu, 2 nymphes.

- SIDAMO :

Adola, 6-66, zébu, $1 \mathrm{~m}$.

Awasa, 3-66, zébu, $3 \mathrm{~m}$.; 5-69, zébu, $1 \mathrm{~m}$.

Bogol Mayo, 5-69, zébu, 8 m., 6 f. 
Degdebe, 1-69, zébu, $1 \mathrm{~m}$., 2 f.

Eloya, 5-69, zébu, 7 m., 2 f.

Filtu, 3-69, zébu, 13 m., 55 f.; 5-69, zébu, $75 \mathrm{~m}$.

Iavello, 5-69, zébu, 4 m., 3 f.

Lei, 5-69, dromadaire, $3 \mathrm{~m}$.

Moyale, 5-69, zébu, 9 m., 4 f.

Negele, 12-68, zébu, 3 m., 2 f.

Paradissa, 5-69, dromadaire, $7 \mathrm{~m}$., zébu, 3 m., $6 \mathrm{f}$.

Tertale, 5-69, zébu, $10 \mathrm{~m}$.

Wachille, 5-68, zébu, $2 \mathrm{~m}$.

\section{- TIGRE :}

Shikat, 4-66, zébu, 4 m., 2 f.

Cercer, 1-67, zébu, 2 m., 2 f.

Abiy Adi, 5-66, zébu, 8 m., 4 f.

A. hebraeum: (Koch, 1844), signalée par E. STELLA, n'a été retrouvée par nous qu'une fois.

\section{- ARUSSI :}

Aboussa Tensai, 4-66, zébu, $1 \mathrm{~m}$.

A. lepidum: (Dönitz, 1909), 129 mâles, 29 femelles.

Quelques exemplaires ont été identifiés par H. HOOGSTRAAL. Cette tique a été retrouvée dans six provinces (carte).

\section{- ERYTHREE :}

Asmara, 12-66, zébu, 12 m., 2 f.

- GEMU GOFA :

Jinka, 2-69, zébu, $12 \mathrm{~m}$.

- HARAR :

Elbah (900 m), chèvre, 3 m, 1 f., dromadaire, 39 m., $5 \mathrm{f}$.

Erer (1 $000 \mathrm{~m}), 7-66$, zébu, $4 \mathrm{~m} ., 14 \mathrm{f}$.

Houri Tolo, 7-66, zébu, 34 m., 1 f.

- SHOA :

Koka, 1-67, zébu, $3 \mathrm{~m}$.

- SIDAMO :

Adola, 6-66, zébu, $1 \mathrm{~m} ., 1 \mathrm{f}$.

Paradissa, 5-66, zébu, $1 \mathrm{~m}$.

- TIGRE :

Abergele, 5-66, zébu, $2 \mathrm{~m}$.

Adi Hagada, 9-66, zébu, 12 m., 5 f.

Cercer, 1-67, zébu, $5 \mathrm{~m}$.

Shikat, 5-66, zébu, 2 m.
A. marmoreum: (Koch, 1844), 27 mâles, 13 femelles.

- ARUSSI :

Lac Zwai, 6-66, tortue, 5 m., 2 f.

- GEMU GOFA :

Tourmi, 6-69, tortue, $2 \mathrm{~m}$.

- SHOA :

Awash, 8-66, tortue, $1 \mathrm{~m} ., 1 \mathrm{f}$.

- SIDAMO :

Awasa, 5-68, tortue, $7 \mathrm{~m}$; 3-69, tortue, $1 \mathrm{~m}$. Bifata, 5-69, tortue, $11 \mathrm{~m} ., 10 \mathrm{f}$.

A. nuttalli : (Dönitz, 1909), 3 mâles.

\section{- ARUSSI :}

Teferi Birhan, 3-67, zébu, $3 \mathrm{~m}$.

Deux de ces tiques furent envoyées à HOOGSTRAAL. Il les a considérées comme très proches de $A$. nuttalli, bien que ce dernier soit principalement un parasite de reptiles infestant rarement les mammifères et les oiseaux.

A. variegatum : (Fabricius, 1794), 1887 mâles, 443 femelles, 5 nymphes (carte).

Très commune sur le bétail dans toute l'Ethiopie, cette tique est le vecteur actif de la cowdriose. Il semble que l'humidité joue un rôle inhibiteur dans sa reproduction; en effet, il nous a été donné d'observer, dans la région de Debre-Zeit, la disparition du parasitisme par cette tique pendant la saison des pluies, soit du 20 juillet au 20 septembre; ce fait a été également noté par l'équipe chinoise dans la région de Makale (Tigre). Enfin, dans une expérience de contrôle faite au laboratoire, des œufs de $A$. variegatum pondus respectivement les 17 et 18 juillet, n'ont éclos que le 10 octobre, c'est-àdire 3 semaines après la saison des pluies.

- ARUSSI :

Aboussa Tensai, 4-66, zébu, 6 m., $4 \mathrm{f}$.

Cofele, 4-69, zébu, 1 m., 1 f.; 5-69, zébu, 4 m., 3 f.

Lac Zwai, 11-66, zébu, $3 \mathrm{~m}$.

Tibela : $1290 \mathrm{~m}), 11-65$, cheval, $10 \mathrm{~m}$., 2 f., zébu, 19 m., $6 \mathrm{f}$.

- BALE :

Dincho, 10-66, mouton, $1 \mathrm{~m}$.

Ginir, 5-69, zébu, $1 \mathrm{~m}$., 5 f. 
Goba, 10-66, hyène, $4 \mathrm{~m}$.

Goro (1 $800 \mathrm{~m})$, 9-68, zébu, $24 \mathrm{~m} ., 18$ f.

Maslo, 10-66, dromadaire, $4 \mathrm{~m}$., zébu, $10 \mathrm{~m}$., 2 f.

\section{- BEGEMDER :}

Adiszemen 11-67, zébu, 17 m., 3 f.

Delache, 6-67, zébu, $1 \mathrm{~m}$.; 11-67, zébu, $1 \mathrm{~m}$.

Gehaba, 11-67, zébu, 3 m., 3 f.

Gondar, 6-67, zébu, 1 m., 1 f.; 11-67 zébu, $3 \mathrm{~m}$; 3-69, zébu, $10 \mathrm{~m}$.

Gorgora, 4-66, zébu, 5 m.; 11-67, zébu, 10 m., 3 f.; 3-69, zébu, $6 \mathrm{~m}$.

Koladiba, 5-66, zébu, 14 m., 1 f.; 11-67, zébu, $17 \mathrm{~m}$., $6 \mathrm{f}$.

Nefas Mewcha, 6-67, zébu, $1 \mathrm{~m}$; 11-67, zébu, 5 m.; 2-69, zébu, 3 f.; 5-69, zébu, 6 f.

\section{- ERYTHREE :}

Asmara, 12-66, zébu, 48 m., 8 f.

\section{- GEMU GOFA :}

Bako, 6-66, zébu, 46 m., 4 f.

Bulki, 6-66, zébu, 5 m., $6 \mathrm{f}$.

Djinka, 5-69, zébu, 21 m., 4 f.

Kayafer, 5-69, zébu, 100 m., 36 f.

Sele, 5-65, zébu, 62 m., 6 f., 3 nymphes.

\section{- GODJAM :}

Bahar dar (2 $000 \mathrm{~m}$ ) 2-68, zébu, 14 m., 3 f.

\section{- HARAR :}

Alemaya, 4-66, zébu, 104 m., 17 f.

Dire Dawa, 4-67, zébu, 10 m., 9 f.

Elbah, 4-67, zébu, 35 m., 20 f.

Erer, 4-67, zébu, 22 m., 5 f.

- ILLUBABOR :

Gore, 5-66, zébu, 2 m.

- KAFFA :

Bonga, 4-66, zébu, 4 m., 1 f.

Jimma, 5-66, zébu, $5 \mathrm{~m}$.

Wako, 5-66, zébu, 25 m., 43 f.

Wushwush, 4-66, zébu, $3 \mathrm{~m}$.

- SHOA :

Adamitulu, 6-67, zébu, 140 m., 5 f.

Alem Tena, 6-67, zébu, $19 \mathrm{~m}$., $3 \mathrm{f}$.

Debre Zeit, 5-66, zébu, 55 m., 16 f.; 6-66, mouton, 8 m., 1 f., zébu, 22 m., 9 f., 7-66, zébu, $41 \mathrm{~m}$.

Endeber, 5-67, âne, 4 m., 1 f., zébu, $101 \mathrm{~m}$. 30 f.; 5-67 cheval, 2 nymphes.
Koka, 6-66, chèvre, 3 m., zébu, $11 \mathrm{~m}$; 6-67, zébu, $42 \mathrm{~m}$.

Mojo, 5-66, zébu, $12 \mathrm{~m}$.

Nazareth, 6-66, chèvre, $1 \mathrm{~m}$.

Shashamane, 2-66, zébu, 11 m., 6 f.; 3-69, zébu, $1 \mathrm{~m} ., 1 \mathrm{f}$.

Sodere 6-66, zébu, $46 \mathrm{~m}$.

- SIDAMO :

Adola, 6-66, zébu, $5 \mathrm{~m}$., $1 \mathrm{f}$.

Awasa, 3-65, zébu, 29 m., 2 f.; 5-65, zébu, $9 \mathrm{~m} ., 14 \mathrm{f}$.

Burji, 5-69, zébu $11 \mathrm{f}$.

Butchisse, 5-65, zébu, $120 \mathrm{~m}$.

Dolo, 4-66, zébu, $1 \mathrm{~m}$.

Gudunu, 5-68, zébu, $2 \mathrm{~m}$., $6 \mathrm{f}$.

Iavello, 6-66, dromadaire, 2 m., zébu, 17 m., $1 \mathrm{f}$.

Idilola 5-66, zébu, 3 m., 15 f.

Kilkile, 4-66, zébu, 12 m., 10 f.

Lei, 5-66, dromadaire, $58 \mathrm{~m}$., $8 \mathrm{f}$.

Moyale, 4-65, zébu, $59 \mathrm{~m}$., $30 \mathrm{f}$.

Negele, 4-65, zébu, 46 m., 1 f.; 4-66, zébu, 47 m., 2 f.

Paradissa, 5-66, zébu, $26 \mathrm{~m}$.

\section{- TIGRE :}

Adi Hagada, 11-66, zébu, 23 m., 9 f.

Anderta, 4-66, zébu, 2 m., 2 f.; 1-67, zébu, $9 \mathrm{~m}$.

Cercer, 1-67, zébu, 3m., 5 f.

Chelokot, 5-66, zébu, 10 m., 1 f.; 6-66, zébu, 9 m., $8 \mathrm{f}$.

Makale, 11-66, zébu $3 \mathrm{~m}$. 1 f.

Shikat, 4-66, zébu, 2 m., 2 f.

Simre, 5-66, zébu, 19 m., 4 f.

- WOLLEGA :

Arjo (3 $000 \mathrm{~m}$ ), 12-65, zébu, $12 \mathrm{~m}$.

Asosa, 6-66, zébu, $10 \mathrm{~m}$., $14 \mathrm{f}$.

Dembidolo, 5-68, zébu, 7 m., 2 f.; 5-69, zébu, $2 \mathrm{~m}$.

Doletti (1 $400 \mathrm{~m}), 5-69$, zébu, $3 \mathrm{~m}$.

Gimbi, 5-69, zébu, $2 \mathrm{~m}$.

Seghe, 12-65, zébu, $8 \mathrm{~m}$.

- WOLLO :

Hayk, 1-67, zébu, $3 \mathrm{~m}$.

Kembolcha, 1-67, zébu, $4 \mathrm{~m}$.

Korem, 1-67, zébu, 5 m., 3 f. 
Genre Aponomma : 8 mâles, 1 femelle.

A. exornatum: (Koch, 1844).

- SHOA :

Debre Zeit, 10-68, varan, 8 m., $1 \mathrm{f}$.

Genre Boophilus : 8 mâles, 26 femelles.

B. decoloratus : (Koch, 1844).

C'est la seule espèce de Boophilus que nous ayons rencontrée. Bien que difficile à récolter, elle fut recensée dans 7 provinces mais il est probable qu'elle est présente dans toute l'Ethiopie. E. STELLA l'avait signalée en 1938. C'est un des vecteurs de Babesia bigemina et Anaplasma marginale.

- BEGEMDER :

Debre Tabor, 10-67, zébu, $1 \mathrm{~m}$.

Gehaba, 10-67, zébu, 1 m., 1 f.

Koladiba, 4-66, zébu, $1 \mathrm{f}$.

- HARAR :

Dire Dawa, 5-67, zébu, 1 m., 2 f.

- ILLUBABOR :

Dagobassa, 12-66, zébu, 4 f.

- SHOA :

Maki, 6-68, zébu, 3 m., 2 f.

- SIDAMO :

Awasa, 3-67, zébu, 13 f.

- TIGRE :

Shikat, 11-66, zébu, 2 f.

- WOLLEGA :

Seghe, 12-65, zébu, 2 m., 1 f.

Genre Haemaphysalis : 72 mâles, 32 femelles.

H. aciculifer : (Warburton, 1913), 7 mâles.

- ARUSSI :

Cofele, 6-66, zébu, $5 \mathrm{~m}$.

- SHOA :

Endeber, 5-67, zébu, $1 \mathrm{~m}$.

- SIDAMO :

Awasa, 5-66, zébu, $1 \mathrm{~m}$.

H. leachii leachii : (Audouin, 1827), 19 mâles, 1 femelle.

C'est dans la ville d'Addis Abeba, le seul vecteur connu de la piroplasmose canine.
- ARUSSI :

Tibila, 7-68, chien, $1 \mathrm{~m}$.

- SHOA :

Addis Abeba, 6-68, chien, $17 \mathrm{~m}$.

Debre Zeit, 4-67, chat, $1 \mathrm{~m} ., 1 \mathrm{f}$.

H. leachii muhsami (Santos Dias, 1954).

- SIDAMO :

Awasa, 5-66, mangouste, $1 \mathrm{~m}$.

H. parmata: (Neumann, 1905), 45 mâles, 31 femelles.

- ARUSSI :

Tibila, 2-65, cheval, 7 m., 6 f., zébu, 10 m., $5 \mathrm{f}$.

- HARAR :

Erer, 7-66, zếbu, 1 m., 2 f.

- SHOA :

Mojo, 6-66, bush buck, 25 m., $15 \mathrm{f}$.

Debre Zeit, 10-66, zébu, 2 m., 3 f.

Genre Hyalomma: 318 mâles, 107 femelles, 1 nymphe.

Ce genre est assez uniformément réparti dans le pays mais cependant plus abondant dans les basses terres.

H. dromedarii : (Koch, 1844), 9 mâles, 2 femelles, 1 nymphe.

- HARAR :

Elbah, 7-66, dromadaire, 3 m., 1 f., 1 nymphe, zébu, $3 \mathrm{~m}$.

- TIGRE :

Shikat, 11-66, zébu, 3 m., 1 f.

H. excavatum: (Koch, 1844), 156 mâles, 30 femelles (carte).

- ARUSSI :

Tibila, 4-66, zébu, $5 \mathrm{~m}$.

- BALE :

Elkere, 7-68, zébu, $10 \mathrm{~m} ., 8 \mathrm{f}$.

Goro, 10-67, zébu, 1 m., 2 f.

- BEGEMDER :

Gondar, 7-67, zébu, $3 \mathrm{~m}$.

- ERYTHREE :

Asmara, 5-66, zébu, 36 m., 1 f. 
- GODJAM :

Debre Markos, 2-68, zébu, $1 \mathrm{~m}$.

- HARAR :

Elbah, 7-66 (900 m), dromadaire, $4 \mathrm{~m}$., zébu, $1 \mathrm{~m}$.

Hai Dora, 7-68, zébu, 6 m., 14 f.

- ILLUBABOR :

Dembi, 7-67, zébu, 24 m., 1 f.

Metu, 9-66, chèvre, 1 m., 2 f.

- SHOA :

Koka, 12-67, zébu, $2 \mathrm{~m}$.

Maki, 6-68, zébu, 3 m., 1 f.

- SIDAMO :

Ayadimtu, 5-69, dromadaire, $21 \mathrm{~m}$.

Filtu, 5-69, zébu, $34 \mathrm{~m}$.

Lei, 5-69, zébu, 1 m., 1 f.

Negele, 4-66, zébu, $3 \mathrm{~m}$.

H. impeltatum : (Schulze et Schlottke, 1930), 25 mâles, 2 femelles.

- BALE :

Goro, 9-68, dromadaire, $1 \mathrm{~m} ., 1 \mathrm{f}$.

Maslo, 8-68, dromadaire, $1 \mathrm{~m}$., zébu, $4 \mathrm{~m}$.

- HARAR :

Hai Dora, 7-66, zébu, $10 \mathrm{~m}$.

Houri Tolo, 7-66, zébu, $9 \mathrm{~m}$.

- TIGRE :

Shikat, 4-66, zébu, 1 f.

H. marginatum: (Koch, 1844), 1 femelle.

- TIGRE :

Shikat, 4-66, zébu, 1 f.

H. rufipes : (Koch, 1844), 55 mâles, 27 femelles (carte).

Cette tique est responsable de la paralysie du mouton.

- ARUSSI :

Tibila, 4-66, zébu, $1 \mathrm{~m}$.

- BEGEMDER :

Gorgora, 3-69, zébu, $3 \mathrm{~m}$.

- HARAR :

Elbah $(900 \mathrm{~m}), 7-66$, dromadaire, $2 \mathrm{~m}$.

Hai Dora, 7-66, zébu, $2 \mathrm{~m}$.

- SHOA :

Koka (1 $550 \mathrm{~m}), 1-67$, zébu, $1 \mathrm{~m}$.
- SIDAMO :

Filtu, 5-69, zébu, 3 m., 3 f.

Wachile, 5-69, zébu, $2 \mathrm{~m}$.

- TIGRE :

Adi Hagada, 10-66, zébu, 17 m., 7 f.

Adiawala, 10-66, zébu, $1 \mathrm{~m}$.

Enderta, 1-67, zébu, 1 m., 1 f.

Makale, 10-66, zébu, 14 m., 11 f.

Samre, 1-67, zébu, 4 m., 1 f.

Shikat, 10-66, zébu, 4 m., 4 f.

H. truncatum: (Koch, 1844), 73 mâles, 45 femelles (carte).

- ARUSSI :

Aboussa Tensai, 4-66, zébu, $2 \mathrm{~m}$.

- BEGEMDER :

Debre Tabor, 10-66, zébu, $7 \mathrm{~m}$.

Gondar, 9-66, zébu, 5 f.

Gorgora, 8-67, zébu, 2 m., 2 f.

- ERYTHREE :

Asmara, 5-66, zébu, $3 \mathrm{~m}$.

- GEMU GOFA :

Jinka, 2-69, zébu, 5 f.

- HARAR :

Dire Dawa, 7-66, zébu, $8 \mathrm{~m} ., 9 \mathrm{f}$.

Elbah, 7-67, dromadaire, $4 \mathrm{~m}$, zébu, $8 \mathrm{~m}$., $2 \mathrm{f}$.

Hai Dora, 7-66, zébu, 10 m., 5 f.

- SHOA :

Addis Abeba, 9-66, mouton, 1 m., zébu, $4 \mathrm{~m}$.

Koka, 8-66, chèvre, 1 m., 1 f., zêbu, 6 m., $1 \mathrm{f}$; 9-66, mouton, $1 \mathrm{~m}$.

Maki, 6-68, zébu, 8 m., $3 \mathrm{f}$.

- SIDAMO :

Ayadimtu 5-69, dromadaire, 3 m., 2 f.

Negele, 4-66, zébu, $3 \mathrm{~m}$.

Wachille, 5-69, zébu, $1 \mathrm{~m}$.

- TIGRE :

Adiawala, 9-66, zébu, $2 \mathrm{~m}$.

Adi Hagada, 9-66, zébu, 4 m., 2 f.

Cercer, 1-67, zébu, 2 m., 1 f.

Genre Rhipicephalus : 3582 mâles, 1491 femelles, 111 nymphes. 
Ce genre est très important en Ethiopie et certaines espèces sont des vecteurs actifs de piroplasmoses et theilerioses.

R. appendiculatus : (Neumann, 1901), 163 mâles, 63 femelles; vecteur de theilerioses et piroplasmoses.

- BALE :

Goba, 5-67, zébu, $28 \mathrm{~m}$.

- BEGEMDER :

Gorgora, 8-67, zébu, $1 \mathrm{~m}$., $1 \mathrm{f}$.

- GEMU GOFA :

Kayafer, 4-67, zébu, 24 m., 2 f.; 11-67, zébu, 5 m., 2 f.

Tourmi : (900 m), 4-67, âne, 11 m., 20 f., zébu, $1 \mathrm{~m}$.

- GODJAM :

Debre Markos 10-67, mouton, 38 m., 14 f.. zébu, 49 m., 24 f.

- HARAR :

Dire Dawa, 5-67, zébu, $2 \mathrm{~m}$.

- SIDAMO :

Butchisse, zébu, $4 \mathrm{~m}$.

R. bursa: (Canestrini et Fanzago, 1878), 3 mâles.

- SHOA :

Addis Abeba, 3-65, chien, 1 m.; 4-66, zébu, $1 \mathrm{~m}$.

- SIDAMO :

Wachile, 5-69, zébu, $1 \mathrm{~m}$.

R. compositus : (Neumann, 1897), 1 mâle, 1 femelle.

- WOLLEGA :

Arjo, 5-69, zébu, 1 m., 1 f.

R. evertsi evertsi : (Neumann, 1897), 143 mâles, 26 femelles, 108 nymphes.

Vecteur de Nuttallia equi et Babesia bigemina (carte).

- ARUSSI :

Cofele, 4-69, zébu, $5 \mathrm{~m}$.

Hoursa (2 $500 \mathrm{~m}$ ), 6-66, mouton, $7 \mathrm{~m} ., 7 \mathrm{f}$.

- BALE :

Goba, 1-67, zébu, 3 m., 1 f., 3 nymphes. Goro (1 $800 \mathrm{~m}$ ), 9-68, zébu, 3 m., 2 f.

Selka, 10-67, zébu, $2 \mathrm{~m}$.

\section{- BEGEMDER :}

Adiszemen, 7-67, zébu, $1 \mathrm{~m} ., 1 \mathrm{f}$.

Debre Tabor, 11-67, zébu, $4 \mathrm{~m}$.

Delache, 11-67, zébu, 8 m., 2 f.

Gehaba, 11-67, zébu, 7 m., 1 f.

Gondar, 4-66, zébu, 6 m., 1 f.; 8-67, zébu,

1 m., 1 f.,; 10-67, zébu 9 m., 2 f.; 11-67,

zébu, $5 \mathrm{~m}$., 1 f.; 5-69, zébu, $7 \mathrm{~m}$.

Gorgora, 3-69, zébu, $6 \mathrm{~m}$.

Koladiba, 5-69, zébu, $6 \mathrm{~m}$.

Mizaba Mariam, 4-66, zébu, $5 \mathrm{~m}$.

- GEMU GOFA :

Sile, 5-69, zébu, $3 \mathrm{~m}$.

- GODJAM :

Debre Markos, 6-67, zébu, 2 m., 1 f.

- HARAR :

Dire Dawa, 5-67, zébu, $1 \mathrm{~m}$.

Elbah, 7-66, chèvre, 3 m., 2 f., mouton,

2 m., 1 f.; 5-67, zébu, 6 m., 2 f.

Houri Tolo, 5-67, zébu, $1 \mathrm{~m}$.

- KAFFA :

Bonga, 4-66, zébu, $3 \mathrm{~m}$.

- SHOA :

Debre Zeit, 10-66, zébu, 1 m.; 3-67, zébu, $1 \mathrm{~m}$; 4-67, zébu, $1 \mathrm{~m}$.

Endeber, 5-67, zébu, $4 \mathrm{~m}$.

Koka, 8-66, zébu, 5 m.; 9-66, mouton, 1 m.; 1-67, zébu, $1 \mathrm{~m}$.; 5-67, zébu, $1 \mathrm{~m}$.

Shashamane, 4-66, zébu, 4 m., 101 nymphes (oreilles).

- SIDAMO :

Arika (1600 m), 6-68, cheval, $4 \mathrm{~m}$.

Awasa, 6-66, zébu, 4 nymphes.

Butchisse, 10-69, zébu, $7 \mathrm{~m}$.

- WOLLEGA :

Gimbi, 5-69, zébu, 1 m., 1 f.

Seghe, 5-69, zébu, $5 \mathrm{~m}$.

R. humeralis : (Rondelli, 1926), (examiné par HOOGSTRAAL), 3 nymphes.

- HARAR :

Houri Tolo, 7-66, zébu, 3 nymphes.

R. kochi : (Dönitz, 1905), 2 mâles.

- SHOA :

Zukala $(3000 \mathrm{~m}), 10-65$, bushbuck, $2 \mathrm{~m}$. 
R. longicoxatus : (Neumann, 1905), 1 mâle. Cette espèce avait été retrouvée en Somalie française par HOOGSTRAAL en 1950 (2).

- HARAR :

Elbah, 7-66, zébu, $1 \mathrm{~m}$.

- SIDAMO :

Wachile, 5-69, zébu, $1 \mathrm{~m}$.

R. longus : (Neumann, 1907), 2 mâles.

- ARUSSI :

Aboussa Tensai, 4-66, zébu, $2 \mathrm{~m}$.

R. oculatus : (Neumann, 1901), 1 mâle.

Emilia STELLA avait signalé cette espèce dans le Harar en 1938.

- HARAR :

Dire Dawa, 10-65, gazelle, $1 \mathrm{~m}$.

R. pravus : (Dönitz, 1910), 224 mâles, 30 femelles (carte).

- BEGEMDER :

Gorgora, 10-65, zébu, $4 \mathrm{~m}$.

- GEMU GOFA :

Arba Minch, 5-69, homme, $1 \mathrm{~m}$.

Jinka, 2-69, zébu, $5 \mathrm{~m}$.

Sile, 5-69, chèvrc, 1 m., 2 f., zébu, $1 \mathrm{~m}$.

- HARAR :

Elbah (900 m), 7-66, chèvre, 12 m.; 5-69, chèvre, $5 \mathrm{~m}$., zébu, $1 \mathrm{~m}$.

Hai Dora (1 $100 \mathrm{~m}$ ), 7-66, zébu, $1 \mathrm{~m}$.

- SHOA :

Debre Zeit, 10-65, zébu, $4 \mathrm{~m}$.

- SIDAMO :

Arika (1 $600 \mathrm{~m}), 5-69$, cheval, $1 \mathrm{~m}$.

Ayadimtu, 5-69, dromadaire, 25 m., 13 f.

Filtu, 5-69, dromadaire, $57 \mathrm{~m}$.

Lei, 5-67, zébu, 112 m., 12 f.

Moyale, 5-69, zébu, $1 \mathrm{f}$.

Wachile, 5-69, zébu, 14 m., 2 f.

R. pulchellus : (Gerstaecker, 1873), 2130 mâles, 984 femelles (carte).

Cette tique, très fréquente dans les régions sèches, ne serait pas un vecteur important (9).

- ARUSSI :

Hoursa (2 $800 \mathrm{~m}), 6-66$, mouton, $3 \mathrm{~m}$, $2 \mathrm{f}$., zébu, $6 \mathrm{~m} ., 6 \mathrm{f}$.

Tibila, 2-65, cheval, $2 \mathrm{~m}$.
- BALE :

Goro, 8-68, dromadaire, 4 m., 1 f., zébu, $1 \mathrm{~m}$.

Maslo, 8-68, zébu, $15 \mathrm{~m} ., 5 \mathrm{f}$.

Selka, 10-67, zébu, 3 m., 3 f.

- BEGEMDER :

Adiszemen, 7-67, zébu, 1 m., 1 f.

Gondar, 4-66, zébu, $1 \mathrm{~m}, 2 \mathrm{f}$.

Gorgora, 4-66, zébu, 9 m., $6 \mathrm{f}$.

Koladiba, 4-66, zébu, $6 \mathrm{~m}$.

- ERYTHREE :

Asmara, 5-66, zébu, 7 m., 6 f.

- GEMU GOFA :

Jinka, 2-69, zébu, $2 \mathrm{~m}$.

Kayafer, 5-68, zébu, $11 \mathrm{~m}$.

Sile, 5-68, zébu, 16 m., $5 \mathrm{f}$.

Turmi, 5-68, âne, 231 m., 51 f., zébu, $155 \mathrm{~m} ., 82 \mathrm{f}$.

- HARAR :

Dire Dawa, 7-66, zébu, 1 m., 2 f.

Elbah (900 m), 7-66, chèvre, 6 m., 2 f., zébu, $7 \mathrm{~m}$., $1 \mathrm{f}$.

Erer, 7-66, zébu, $24 \mathrm{~m}$, $18 \mathrm{f}$.

- SHOA :

Awash, 5-69, zébu, 8 m., 2 f.

Debre Zeit, 4-67, zébu, $11 \mathrm{~m}$.

Koka, 8-66, chèvre, 1 m., 9-96, mouton, 1 m.; 1-67, zébu, 1 m.; 3-67, zébu, 5 m.

\section{- SIDAMO :}

Adola, 6-66, mouton, 2 m., 5 f., zébu, 13 m., $5 \mathrm{f}$.

Ayadimtu, 5-69, dromadaire, 2 m., 2 f.

Bogol Mayo, 5-69, zébu, 36 m., 39 f.

Butchisse, 10-69, zébu, $28 \mathrm{~m} ., 8 \mathrm{f}$.

Eloya, 5-69, zébu, $141 \mathrm{~m} ., 46 \mathrm{f}$.

Filtu, 5-69, dromadaire, 738 m., 374 f., zébu, 327 m., $248 \mathrm{f}$.

Kilkile, 5-69, zébu, 1 m., 1 f.

Lei, 5-69, dromadaire, 118 m., 3 f., zébu, $1 \mathrm{~m}$.

Moyale, 5-69, zébu, 46 m., 27 f.

Negele, 12-68, zébu, $81 \mathrm{~m} ., 18 \mathrm{f}$.

Paradissa, 5-69, zébu, $11 \mathrm{~m}$.

Tertale, 5-69, zébu, $28 \mathrm{~m} ., 9 \mathrm{f}$.

Wachille, 12-68, dromadaire, 3 m.; 5-69, dromadaire, 2 m., 1 f., zébu, 1 m., 2 f. 


\section{- TIGRE :}

Cercer, 1-67, zébu, $3 \mathrm{~m}$.

Enderta, 11-66, zébu, 7 m., 3 f.

R. sanguineus : (Latreille, 1806), 20 mâles, 5 femelles (carte).

Vecteur des piroplasmoses, anaplasmoses et rickettsioses.

- BALE :

Selka, 10-67, zébu, $1 \mathrm{~m}$.

- BEGEMDER :

Gorgora, 10-65, zébu, $1 \mathrm{~m}$.

\section{- HARAR :}

Dire Dawa, 5-67, zébu, $1 \mathrm{~m}$.

- SHOA :

Addis Abeba, 9-68, zébu, $2 \mathrm{~m} ., 1 \mathrm{f}$.

Debre Zeit, 8-65, chien, $1 \mathrm{~m}$., zébu, $1 \mathrm{~m}$.

- SIDAMO :

Awasa, 6-68, zébu, $1 \mathrm{~m} ., 1 \mathrm{f}$.

- TIGRE :

Adi Hagada, 10-66, zébu, 2 m., $1 \mathrm{f}$.

Samre, 1-67, zébu, $9 \mathrm{~m}$., $1 \mathrm{f}$.

Shikat, 7-66, zébu, $1 \mathrm{~m} ., 1 \mathrm{f}$.

R. simus simus : (Koch, 1844), 667 mâles, 339 femelles (carte).

Vecteur d'Anaplasma marginale et Babesia bigemina.

\section{- ARUSSI :}

Asella, 3-68, zébu, 2 f.

Lac Zwai, 10-65, phacochère, $2 \mathrm{~m}$.

Tibila, 7-68, chien, $5 \mathrm{~m}$., $15 \mathrm{f}$, homme, $1 \mathrm{~m}$., $4 \mathrm{f}$.

- BALE :

Goba, 4-67, zébu, 2 m., 2 f.

Goro, 9-68, dromadaire, $1 \mathrm{~m} ., 1 \mathrm{f}$.

Selka, 10-67, zébu, $1 \mathrm{~m}$.

\section{- BEGEMDER :}

Gondar, 4-67, zébu, 2 m., 1 f.

Gorgora, 6-67, zébu, 1 f.; 8-67, zébu, $1 \mathrm{~m}$.

- ERYTHREE :

Asmara, 5-66, zébu, $1 \mathrm{f}$.

- GEMU GOFA :

Jinka, 2-69, zébu, 1 m., 2 f.

Kayafer, 4-67, zébu, $1 \mathrm{~m}$.

Sile, 5-69, zébu, $2 \mathrm{~m}$.
- HARAR :

Alemaya, 4-67, hyène, $9 \mathrm{~m}$., $3 \mathrm{f}$.

Dire Dawa, 3-68, zébu, $4 \mathrm{~m}$.

Elbah $(900 \mathrm{~m}), 7-66$, chèvre, $5 \mathrm{f}$.

Hai Dora, 7-66, zébu, $11 \mathrm{~m}$.

- KAFFA :

Jimma, 7-69, zébu, 3 f.

- SHOA :

Debre Zeit, 9-65, chien, 1 m.; 12-66, chien, $1, \mathbf{m}$.

Koka, 8-66, chèvre, $7 \mathrm{~m}$., $1 \mathrm{f}$., porc, $25 \mathrm{~m}$., 3 f., zébu, $18 \mathrm{~m}$., 2 f.; 9-66, mouton, 8 m.; 11-67, mouton, 11 m., 1 f., zébu, 4 m., 2 f.

- SIDAMO :

Ayadimtu, 5-69, dromadaire, 222 m., 134 f.

Awasa, 8-68, zébu, $7 \mathrm{~m} ., 4 \mathrm{f}$.

Dila, 11-66, zébu, $1 \mathrm{~m}$.

Filtu, 5-69, dromadaire, 98 m., 63 f., zébu, 68 m., 7 f.

Kersabule, 7-66, dromadaire, 43 f.; 3-69, dromadaire, $57 \mathrm{~m}$.

Lei, 5-69, dromadaire, 5 f., zébu, $10 \mathrm{~m}$., $23 \mathrm{f}$.

Wachille, 12-68, zébu, 6 m.; 5-69, dromadaire, $6 \mathrm{~m}$., zébu, $85 \mathrm{~m}$., $10 \mathrm{f}$.

- TIGRE :

Abergele, 7-66, zébu, $3 \mathrm{~m}$.

Adi Hagada, 10-66, zébu, $1 \mathrm{~m}$., $1 \mathrm{f}$.

Cercer, 11-66, zébu, 1 m.; 1-67, zébu, $1 \mathrm{~m}$.

R. sulcatus: (Neumann, 1908), 12 mâles, 5 femelles.

- BEGEMDER :

Gondar, 7-66, zébu, 3 m., 1 f.

- GODJAM :

Debre Markos, zébu, 1 m., $1 \mathrm{f}$.

- HARAR :

Alemaya, 10-67, hyène, 8 m., $3 \mathrm{f}$.

R. supertritus: (Neumann, 1907), 14 mâles, 7 femelles.

- HARAR :

Alemaya, 4-67, hyène, $14 \mathrm{~m} ., 7 \mathrm{f}$.

R. tricuspis : (Dönitz, 1906), 75 mâles, 5 femelles.

- BALE :

Goba, 5-67, cheval, $1 \mathrm{~m}$. 


\section{BEGEMDER :}

Delache, 6-67, chien, $1 \mathrm{~m} ., 2 \mathrm{f}$.

Gehaba, 6-67, zébu, $2 \mathrm{~m}$.

Gondar, 9-66, zébu, 3 m.; 7-67, zébu, 8 m.; 11-67, zébu, $2 \mathrm{~m}$.

\section{- KAFFA :}

Jimma, 5-66, zébu, 3 m., 1 f.; 7-69, zébu, 2 m., $1 \mathrm{f}$.

\section{- SHOA :}

Debre Zeit, 9-66, zébu, $1 \mathrm{~m}$.

- SIDAMO :

Negele, 5-69, zébu, $50 \mathrm{~m}$.

R. turanicus: (Pomernacev et Matikasvili, 1940), 124 mâles, 26 femelles.

- GODJAM :

Debre Markos, 2-68, zébu, 85 m., 8 f .
- GEMU GOFA :

Bulki, 2-69, zébu, 19 m., 7 f.

- SIDAMO :

Adola, 5-69, zébu, 20 m., 11 f.

Cet inventaire n'a pas la prétention d'être complet : en effet, ainsi qu'il fut déjà signalé, les prélèvements portent avant tout sur le bétail, la faune sauvage ayant été d'accès difficile. Cependant, si quelques espèces restent encore à trouver, leur méconnaissance ne peut guère influencer fortement les résultats obtenus et l'exploitation de ces derniers permet de préciser la fréquence ainsi que la répartition de nombreuses tiques courantes susceptibles d'être de dangereux vecteurs actifs.

Un classement essentiellement quantitatif (tableau $\mathrm{n}^{\circ} \mathrm{I}$ ), ne tenant pas compte des hôtes et

TABLEAU No I

\begin{tabular}{|c|c|c|}
\hline Espèce & $\begin{array}{l}\text { Nombre } \\
\text { d'exemplaires } \\
\text { récoltés }\end{array}$ & Pourcentage \\
\hline 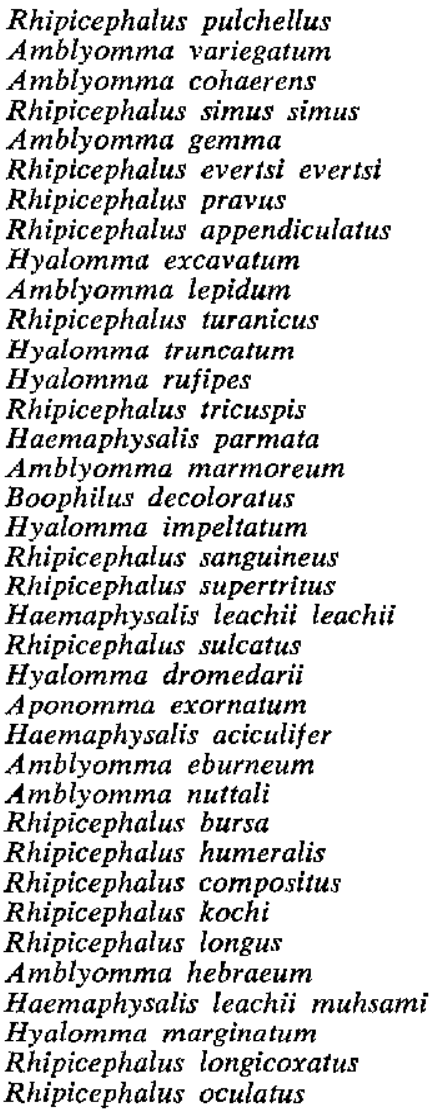 & $\begin{array}{r}3114 \\
2335 \\
1599 \\
1006 \\
338 \\
277 \\
254 \\
226 \\
186 \\
158 \\
150 \\
118 \\
82 \\
80 \\
76 \\
40 \\
34 \\
27 \\
25 \\
21 \\
20 \\
17 \\
12 \\
9 \\
7 \\
5 \\
3 \\
3 \\
3 \\
2 \\
2 \\
2 \\
1 \\
1 \\
1 \\
1 \\
1\end{array}$ & $\begin{array}{r}30,50 \\
22,80 \\
15,63 \\
9,83 \\
3,30 \\
2,70 \\
2,48 \\
2,20 \\
1,81 \\
1,54 \\
1,46 \\
1,15 \\
0,80 \\
0,77 \\
0,73 \\
0,39 \\
0,33 \\
0,26 \\
0,24 \\
0,20 \\
0,19 \\
0,16 \\
0,12 \\
0,09 \\
0,07 \\
0,05 \\
0,03 \\
0,03 \\
0,03 \\
0,02 \\
0,02 \\
0,02 \\
0,01 \\
0,01 \\
0,01 \\
0,01 \\
0,01\end{array}$ \\
\hline
\end{tabular}


des régions met en valeur 4 espèces :

Rhipicephalus pulchellus,

Amblyomma variegatum,

Amblyomma cohaerens,

Rhipicephalus simus simus.

Cependant, ceci ne reflète pas tout à fait la réalité car Rhipicephalus pulchellus, par exemple, fut surtout trouvé dans des régions sèches et spécialement dans le Sidamo.
Un autre classement quantitatif portant sur 7 espèces animales domestiques (tableau $\mathrm{n}^{\circ} \mathrm{II}$ ), bien que ne tenant pas compte des régions, fait cependant ressortir chez le zébu :

Amblyomma variegatum,

Amblyomma cohaerens,

Rhipicephalus pulchellus,

la première de ces tiques étant spécialement dangereuse par son rôle bien connu dans la cowdriose.

TABLEAU No II

\begin{tabular}{|c|c|c|c|c|c|}
\hline & $\begin{array}{l}\text { Nombre } \\
\text { de tiques }\end{array}$ & p. 100 & & $\begin{array}{l}\text { Nombre } \\
\text { de tiques }\end{array}$ & p. 100 \\
\hline \multicolumn{3}{|l|}{ Ane } & \multicolumn{3}{|l|}{ Mouton } \\
\hline R. pulchellus & (282) & 87,6 & $R$. appendiculatus & (52) & 48,6 \\
\hline R. appendiculatus & (31) & 9,6 & R. simus & 20 & 18,7 \\
\hline A. cohaerens & 5 & 1,5 & R. evertsi & 18 & 16,8 \\
\hline A. variegatum & & \multirow[t]{3}{*}{1,3} & R. pulchellus & 11 & 10,5 \\
\hline \multirow{2}{*}{ Cheval } & & & H. truncatum & 2 & 1,37 \\
\hline & & & A. variegatum & 1 & 0,93 \\
\hline A. variegatum & 14 & 36,8 & A. cohaerens & 1 & 0,93 \\
\hline $\begin{array}{l}H . \text { parmata } \\
R . \text { evertsi }\end{array}$ & $\begin{array}{r}13 \\
4\end{array}$ & $\begin{array}{l}34,2 \\
10,5\end{array}$ & A. gemma & 1 & 0,93 \\
\hline A. cohaerens & 3 & 7,9 & A. eburneum & 1 & 0,93 \\
\hline R. pulchellus & 2 & 5,3 & & & \\
\hline R. pravus & 1 & 2,65 & \multirow{2}{*}{\multicolumn{3}{|c|}{ Zébu }} \\
\hline $\boldsymbol{R}$. appendiculatus & 1 & 2,65 & & & \\
\hline \multirow{2}{*}{\multicolumn{3}{|c|}{ Chèvre }} & A. variegatum & 2236 & 30,2 \\
\hline & & & A. cohaerens & 1590 & 21,5 \\
\hline R. pravus & 12 & 24 & R. pulchellus & 1553 & 21 \\
\hline R. simus & 12 & 24 & R. simus & 314 & 4,24 \\
\hline R. pulchellus & 8 & 16 & A. gemma & 312 & 4,21 \\
\hline$R$. evertsi & 5 & 10 & $R$. evertsi & 250 & 3,38 \\
\hline A. lepidum & 4 & 8 & $R$. pravus & 179 & 2,42 \\
\hline $\begin{array}{l}\text { A. variegatum } \\
H . \text { excavatum }\end{array}$ & $\begin{array}{l}3 \\
3\end{array}$ & $\begin{array}{l}6 \\
6\end{array}$ & H. excavatum & 158 & 2,13 \\
\hline $\begin{array}{l}\text { H. excavatum } \\
\text { A. eburneum }\end{array}$ & $\frac{3}{2}$ & $\begin{array}{l}0 \\
4\end{array}$ & R. turanicus & 150 & 2,03 \\
\hline \multirow[t]{2}{*}{ H. truncatum } & \multirow[t]{4}{*}{1} & \multirow[t]{4}{*}{2} & R. appendiculatus & 143 & 1,93 \\
\hline & & & A. lepidum & 110 & 1,49 \\
\hline Chien & & & H. truncatum & 106 & 1,43 \\
\hline & & & H. rufipes & 80 & 1,07 \\
\hline H. leachii leachii & 18 & 72 & R. tricuspis & 76 & 1,01 \\
\hline R. appendiculatus & 3 & 12 & B. decoloratus & 34 & 0,46 \\
\hline R. simus & 2 & 8 & H. impeltatum & 24 & 0,33 \\
\hline R. sanguineus & 1 & 4 & R. sanguineus & 24 & 0,33 \\
\hline \multirow[t]{2}{*}{ R. bursa } & \multirow[t]{3}{*}{1} & \multirow[t]{3}{*}{4} & H. parmata & 23 & 0,32 \\
\hline & & & H. aciculifer & 7 & 0,09 \\
\hline Dromadaire & & & H. dromedarii & 7 & 0,09 \\
\hline R. pulchellus & 1248 & 58 & R. sulcatus & 6 & 0,07 \\
\hline R. simus & 630 & 29,2 & A. nuttali & 3 & 0,04 \\
\hline R. pravus & 95 & 4,4 & $R$. bursa & 3 & 0,04 \\
\hline A. variegatum & 72 & 3,34 & R. humeralis & 3 & 0,04 \\
\hline A. lepidum & 44 & 2,04 & A. eburneum & 2 & 0,03 \\
\hline A. gemma & 25 & 1,16 & R. longicoxatus & 2 & 0,03 \\
\hline H. excavatum & 25 & 1,16 & R. longus & 2 & 0,03 \\
\hline $\begin{array}{l}H . \text { truncatum } \\
H .\end{array}$ & $\begin{array}{l}9 \\
5\end{array}$ & $\begin{array}{l}0,4 \\
0,2\end{array}$ & $R$. compositus & 2 & 0,03 \\
\hline H. impeliatum & 3 & $\begin{array}{l}0,2 \\
0,14\end{array}$ & A. marginatum & 1 & 0,02 \\
\hline H. rufipes & 2 & 0,1 & A. hebraeum & 1 & 0,02 \\
\hline
\end{tabular}

Les chiffres entre parenthèses n'ont pas de valeur statistique car la récolte ne porte que sur un seul individu. 
Chez le dromadaire, dont l'aire géographique est limitée aux régions sèches, nous trouvons en tête de liste Rhipicephalus pulchellus et Rhipicephalus simus simus.

Les petits ruminants, l'âne, le cheval et le chien, ne font pas l'objet de récoltes assez abondantes pour en tirer des conclusions exploitables.

Une répartition qualitative des tiques par province, modifie quelque peu les conclusions précédentes et permet de se rendre compte de l'aire d'extension des différentes espèces: Amblyomma variegatum est présente dans les 14 provinces d'Ethiopie. Mais si Amblyomma cohaerens et Rhipicephalus simus simus sont encore en bonne place, on constate que Rhipicephalus evertsi evertsi a une diffusion aussi importante que les deux précédentes et que Rhipicephalus pulchellus n'est présent que dans 9 provinces. On peut remarquer enfin que certaines espèces, quantitativement discrètes, ont une aire d'extension non négligeable.

\section{CONCLUSION}

$\mathrm{Au}$ total 37 espèces de la famille des Ixodidae et 3 de la famille des Argasidae ont été signalées en Ethiopie. Bien que portant sur

TABLEAU No III

\begin{tabular}{|c|c|c|c|c|c|c|c|c|c|c|c|c|c|c|c|}
\hline & $\begin{array}{l}\text { 莺 } \\
\text { 焉 }\end{array}$ & $\begin{array}{l}\stackrel{0}{0} \\
\frac{8}{5}\end{array}$ & 余 & $\begin{array}{l}\text { 要 } \\
\text { 案 }\end{array}$ & 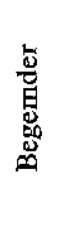 & 㟧 & 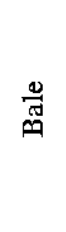 & 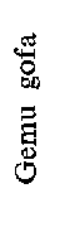 & $\begin{array}{l}\text { 题 } \\
\text { 号 } \\
\end{array}$ & 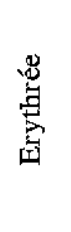 & 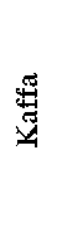 & 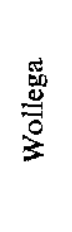 & $\begin{array}{l}\text { वे } \\
\text { 总 } \\
\text { 靣 }\end{array}$ & $\begin{array}{l}\stackrel{9}{\circ} \\
\text { B }\end{array}$ & 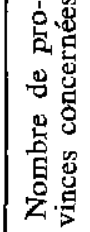 \\
\hline 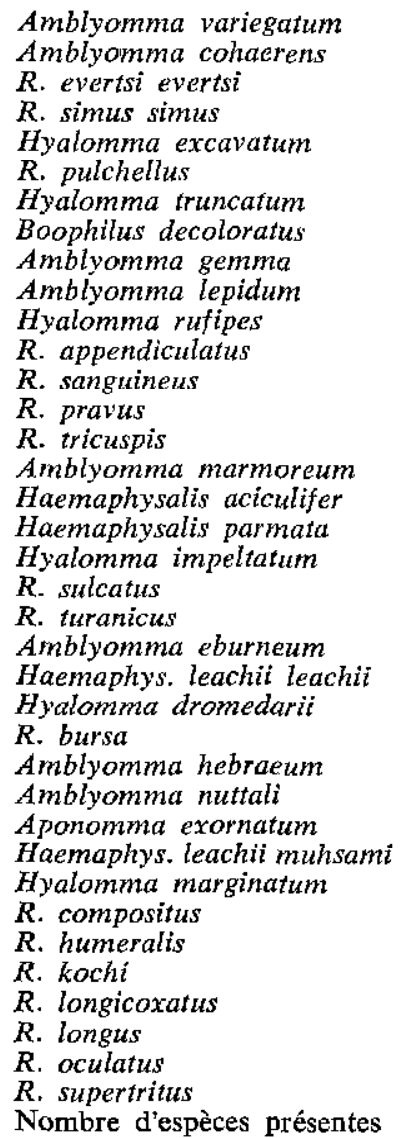 & $\begin{array}{l}+ \\
+ \\
+ \\
+ \\
+ \\
+ \\
+ \\
+ \\
+ \\
+ \\
+ \\
+ \\
+ \\
+\end{array}$ & $\begin{array}{l}+ \\
+ \\
+ \\
+ \\
+ \\
+ \\
+ \\
+ \\
+ \\
+ \\
+ \\
+ \\
+ \\
+ \\
+ \\
+ \\
+ \\
+\end{array}$ & $\begin{array}{l}+ \\
+ \\
+ \\
+ \\
+ \\
+ \\
+ \\
+ \\
+ \\
+ \\
+ \\
+ \\
+ \\
+ \\
+ \\
+ \\
+ \\
+\end{array}$ & $\begin{array}{l}+ \\
+ \\
+ \\
+ \\
+ \\
+ \\
+\end{array}$ & $\begin{array}{l}+ \\
+ \\
+ \\
+ \\
+ \\
+ \\
+ \\
+ \\
+ \\
+ \\
+ \\
+ \\
+\end{array}$ & $\begin{array}{l}+ \\
+ \\
+ \\
+ \\
+ \\
+ \\
+ \\
+ \\
+\end{array}$ & $\begin{array}{l}+ \\
+ \\
+ \\
+ \\
+ \\
+ \\
+ \\
+ \\
+ \\
+ \\
+\end{array}$ & $\begin{array}{l}+ \\
+ \\
+ \\
+ \\
+ \\
+\end{array}$ & $\begin{array}{l}+ \\
+ \\
+ \\
+\end{array}$ & $\begin{array}{l}+ \\
+ \\
+ \\
+ \\
+ \\
+ \\
+\end{array}$ & $\begin{array}{l}+ \\
+ \\
+ \\
+\end{array}$ & $\begin{array}{l}+ \\
+ \\
+\end{array}$ & $\begin{array}{l}+ \\
+ \\
+\end{array}$ & + & $\begin{array}{r}14 \\
10 \\
10 \\
10 \\
9 \\
9 \\
8 \\
7 \\
6 \\
6 \\
6 \\
6 \\
6 \\
5 \\
5 \\
4 \\
3 \\
3 \\
3 \\
3 \\
3 \\
2 \\
2 \\
2 \\
2 \\
1 \\
1 \\
1 \\
1 \\
1 \\
1 \\
1 \\
1 \\
1 \\
1 \\
1\end{array}$ \\
\hline
\end{tabular}


10246 échantillons, cet inventaire est incomplet car ce n'est qu'occasionnellement que nous fûmes amenés à déterminer des tiques prélevées sur les animaux sauvages. Les collectes furent avant tout effectuées sur les espèces domestiques et spécialement sur le gros bétail.

On peut considérer qu'Amblyomma variegatum est à la fois la tique la plus répandue et la plus dangereuse. Rhipicephalus pulchellus et Amblyomma cohaerens n'ont qu'une importance statistique puisqu'on ne les considère pas comme des vecteurs actifs. Par contre, Rhipicephalus simus simus et Rhipicephalus evertsi evertsi ont une aire de répartition assez étendue et sont connues pour transmettre les piroplasmoses, theilerioses et anaplasmoses.

\section{SUMMARY}

\section{Contribution to the study of ticks distribution in the Ethiopian Empire}

Investigation carried out from 1965 to 1969 , the authors studied ticks distribution in Ethiopia and 40 species were inventorized: Rhipicephalus pulchellus, Amblyomma variegatum, Amblyomma cohaerens et Rhipicephalus simus simus, are the most important numerically.

Amblyomma variegatum is the most prevalent species.

\section{RESUMEN}

Contribución al estudio de la repartición de las garrapatas en Etiopia. Encuesta efectuada de 1965 a 1969

Los autores estudiaron la repartición de las garrapatas en Etiopia e hicieron inventario de 40 especies.

Rhipicephalus pulchellus, Amblyomma variegatum, Amblyomma cohaerens y Rhipicephalus simus simus son las más numerosas. Amblyomma variegatum es la especie más encontrada.

\section{BIBLIOGRAPHIE}

1. BERGEON (P.). Report to the Government of Ethiopia on a parasitology survey. Rome, F.A.O., 1968 (No TA. 2458 TE/AN/EIH).

2. HOOGSTRAAL (H.). On ticks (Ixodidae) of southern french Somaliland and the rediscovery of Rhipicephalus longicoxatus (Neumann 1905). Ann. Entomol. Soc. Am., 1953, 46 (3) : 393-398.

3. HOOGSTRAAL (H.). African Ixodoidea. 1. Ticks of the Sudan. Cairo, Egypte, 1958, no 3 (Research report NM 005050.29.07).

4. HOOGSTRAAL (H.). Evidence of rickettsial disease agents from ethiopian cattle. Bull. Org. mond. Santé. 1966, 37 : 127-131.

5. MOREL (P. C.), VASSILIADES (G.). Les Rhipicephalus du groupe sanguineus, espèces africaines. Rev. Elev. Méd. vét. Pays trop., 1962, 15 (4) : 343-385.

6. ROUSSELOT (R.). Notes de parasitologie tropi- cale. Tome 1: parasites du sang des animaux. Tome 2: Ixodes. Paris, Vigot frères, 1953.

7. STELLA (E.). Gli Ixodidi dell'Africa Orientale Italiana. Riv. Biol. Colon., 1938 (1) : 135-153.

8. TOUMANOFF (C.). Les tiques (Ixodoidea) de l'Indochine. Institut Pasteur de l'Indochine SILI Saïgon 1944.

9. WALKER (Jane B.). Rhipicephalus pulchellus Gerstäcker 1873: a description of the larva and nymph with notes on the adults and on its biology. Parasitology, 1955, 45 (1-2) : 92-98.

10. WALKER (Jane B.). Rhipicephalus pravus Dönitz 1910. Parasitology, 1956, 46 (3-4) : 243-260.

11. WALKER (Jane B.). Rhipicephalus humeralis Rondelli 1926. Parasitology, 1957, 47 (1-2): 145 152.

12. WALKER (Jane B.). Notes on the common tick species of East Africa. Nairobi (Box 596), Cooper, Mc Dougall and Robertson (EA) Ltd, 1966. 